\title{
Avalanche Photodiodes and Vacuum Phototriodes for the Electromagnetic Calorimeter of the CMS experiment at the Large Hadron Collider
}

\author{
Peter R Hobson ${ }^{* 1}$
}

School of Engineering \& Design, Brunel University, Uxbridge UB8 3PH, UK

\begin{abstract}
The homogeneous lead tungstate electromagnetic calorimeter for the Compact Muon Solenoid detector at the Large Hadron Collider operates in a challenging radiation environment. The central region of the calorimeter uses large-area avalanche photodiodes to detect the fast blue-violet scintillation light from the crystals. The high hadron fluence in the forward region precludes the use of these photodiodes and vacuum phototriodes are used in this region. The constructional complexity of the calorimeter, which comprises 75848 individual crystals, plus the activation of material make repair during the lifetime of the detector virtually impossible. We describe here the key features and performance of the photodetectors and the quality assurance procedures that were used to ensure that the proportion of photodetectors that fail over the lifetime of CMS will be limited to a fraction of a percent.
\end{abstract}

Electromagnetic calorimetry; Photodetector; Avalanche photodiode; Vacuum phototriode; Radiation hardness

\footnotetext{
${ }^{*}$ Corresponding author. Tel. +44-1895-266799; fax: +44-1895-272391; e-mail: Peter.Hobson@brunel.ac.uk.

${ }^{1}$ On behalf of the CMS ECAL Group.
} 


\section{Introduction}

The Compact Muon Solenoid (CMS) [1] is a general purpose detector at the CERN LHC. A key design feature is the high-resolution homogeneous electromagnetic calorimeter (ECAL), with 75848 scintillating crystals covering almost $4 \pi$ of solid angle. The ECAL must be fast, have high granularity and be radiation resistant to lifetime doses amounting to $4 \mathrm{kGy}$ and $2 \times 10^{13} \mathrm{n} / \mathrm{cm}^{2}$ in the central Barrel and up to 50 times higher in the forward Endcaps. The design of the ECAL is described in detail elsewhere [2].

The active medium of the ECAL is the fast, radiation tolerant scintillator lead tungstate $\left(\mathrm{PbWO}_{4}\right)$ [3]. This produces about $1 \%$ of the light of $\mathrm{NaI}(\mathrm{Tl})$ and emits $80 \%$ of its light within 25 ns in the blueviolet region of the spectrum. The entire calorimeter is inside the $4 \mathrm{~T}$ magnetic field of the CMS solenoid magnet thus the requirement is for radiation tolerant photodetectors that are fast, blue-sensitive and have gain at high magnetic fields.

\section{Photodetectors}

In the Barrel section, the chosen photo-detectors are S8148 avalanche photodiodes [4], developed by Hamamatsu Photonics for CMS. They are almost insensitive to shower leakage particles traversing them. The vacuum phototriodes are single gain-stage, photomultipliers, developed for CMS by RIE, St. Petersburg [5].

The radiation environment within CMS consists of increasing electron/gamma dose and hadron fluencies going from the central region towards the forward as one approaches small angles to the proton beams. The intense hadron flux in the forward region forces a change in technology from silicon avalanche photodetectors to vacuum phototriodes.

\subsection{Avalanche Photodiodes}

The silicon avalanche photodiodes (APD) are $5 \times 5 \mathrm{~mm}^{2}$ in area, with $75 \%$ quantum efficiency (QE) at $\lambda=420 \mathrm{~nm}$ and in CMS are operated at a gain of 50 with an excess noise factor of 2 . The Hamamatsu type S8148 consists of successive layers of $\mathrm{p}^{+}, \mathrm{p}, \mathrm{n}, \mathrm{n}^{-}$ and $\mathrm{n}^{+}$-type silicon layers (figure 1 ).

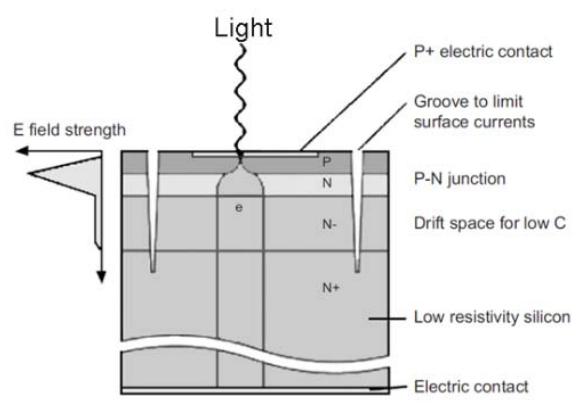

Fig. 1. Schematic diagram of the Hamamatsu S8148 APD

The $\mathrm{p}-\mathrm{n}$ junction of the device, where the avalanche gain takes place, is located around $5 \mu \mathrm{m}$ from where the scintillation light enters the surface to minimize the sensitivity to ionizing radiation. The $\mathrm{n}^{-}$ layer increases the thickness of the depletion region. This decreases both the capacitance and the dependence of the gain on the applied bias voltage. $\mathrm{V}$-shaped grooves, around the cathode reduce surface leakage currents; this is especially important after the device has received a large radiation dose. The properties and working principles of the structure are given in [6]; the variation of gain with bias voltage is shown in figure 2 [7]. The uniformity of the gain across the active area of the device, at the CMS operating gain of 50 , is extremely good indicating excellent doping uniformity and a low level of lattice defects [8].

Every barrel crystal has two APD on its end-face to increase the ratio of photodetector area to that of the crystal.

\subsubsection{Quality Assurance}

To ensure the reliability over the lifetime of the detector, every APD was screened by ${ }^{60} \mathrm{Co}$ irradiation and burned-in before it was accepted for CMS. The following procedure was used to screen each APD for radiation hardness and reliability:

APDs were rejected mainly if their breakdown voltage changed by more than $5 \mathrm{~V}$, or their dark current behaviour as a function of bias voltage was anomalous after irradiation or burn-in, or if their noise was anomalously high after irradiation. 


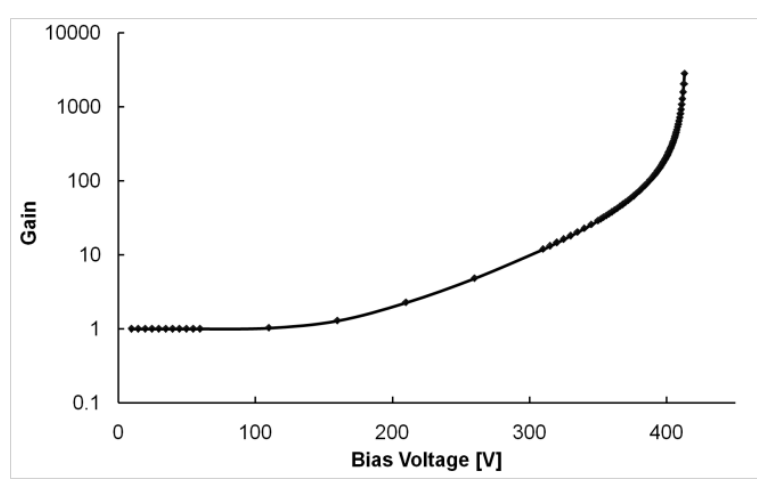

Fig. 2. Variation of gain with bias voltage for a typical Hamamatsu S8148 APD

Testing showed that most APDs suffered no significant change in optical or electrical properties under irradiation, with the exception of an unavoidable increase in dark current (typically $3 \mathrm{nA}$ unirradiated at the operating gain of 50). From the detailed performance studies and $100 \%$ acceptance testing we may thus conclude that the APDs installed in the CMS ECAL should be reliable at the required scale of $99.9 \%$ [9].

\subsection{Vacuum Phototriodes}

APDs are unsuitable for use in the Endcaps, where the gamma and neutron radiation levels are very much higher than in the barrel. For this region specially developed vacuum phototriodes (VPT) were produced by RIE. These single gain-stage photomultipliers have an active area of $280 \mathrm{~mm}^{2}$, with a bialkali photocathode (figure 3). The main radiation damage to the VPT is a darkening (induced absorption peak at $320 \mathrm{~nm}$ ) of the transparent window on which the photocathode is deposited. We identified a radiation resistant UV transparent borosilicate glass which was more cost-effective for this application than fused silica [10]. The VPT is fast, with rise and fall times of order 1ns (figure 4), and has low inter-electrode capacitances of about 3pF.
We operate the VPT with an anode voltage of 1000 , a dynode voltage of 800 and with the photocathode at ground potential. With these parameters the gain at $15^{\circ}$ to the $4 \mathrm{~T}$ field is around 10 and we are insensitive to small variations in the electrode potentials.

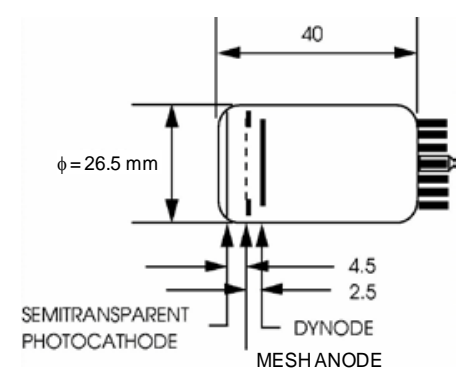

Fig. 3. Schematic of the RIE VPT. The mesh anode is $50 \%$ transparent and has a pitch of $100 \mathrm{~mm}^{-1}$.

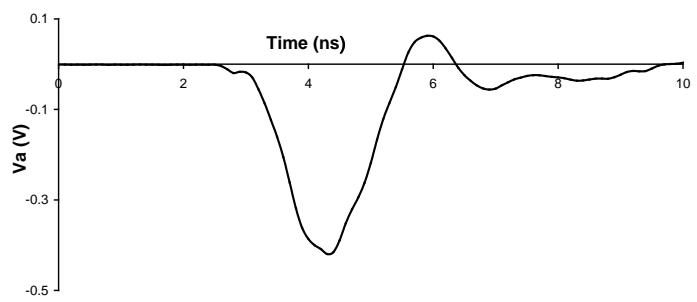

Fig. 4. Average of 2000 anode pulses into 50 ohms from a VPT illuminated by a laser operating at $435 \mathrm{~nm}$ with 80 ps pulse width. The oscilloscope bandwidth was $3 \mathrm{GHz}$ and the repetition rate was $0.8 \mathrm{~Hz}$.

\subsubsection{Quality Assurance}

All of the VPT produced for CMS were re-tested on delivery at various angles in a $1.8 \mathrm{~T}$ magnetic field. Twelve percent of the total were also batch tested at a fixed angle of 15 degrees in a 4T field. To be accepted we required $>15 \%$ QE at $420 \mathrm{~nm}$ and gain $>7$. Figure 5 shows the normalised yield (product of gain and QE) converted to electrons per $\mathrm{MeV}$ deposited in an endcap crystal. 


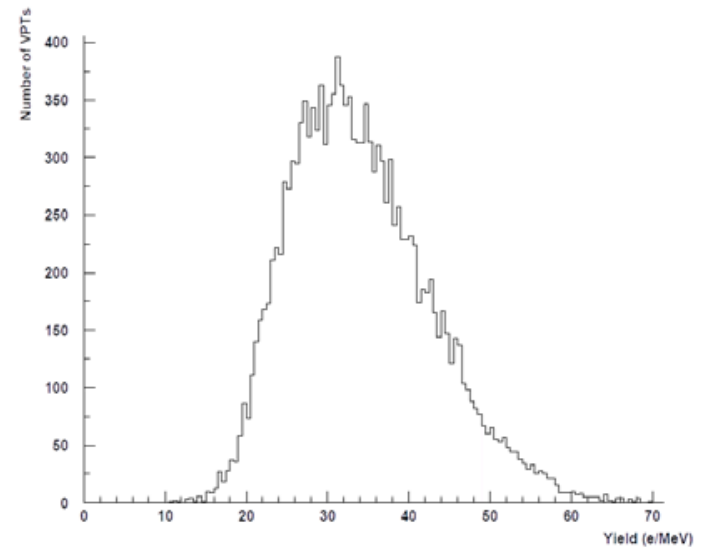

Fig. 5. Normalised yield of production VPT

The response in the $4 \mathrm{~T}$ magnetic field was required to be better than $75 \%$ compared to no magnetic field. No VPT passing the acceptance tests at $1.8 \mathrm{~T}$ failed the $4 \mathrm{~T}$ ratio criterion. Complete VPTs are radiation hard and their response, attributable entirely to the damage to the window, decreases by less than $10 \%$ after $20 \mathrm{kGy}$. Radiation tolerance of all VPT was assured by rigorous testing of sample windows from each batch of glass before production.

\section{Energy Resolution of Barrel ECAL}

Beam tests carried out on barrel modules using production APDs have demonstrated that the detector performance is sufficient to detect the Higgs particle in the $\mathrm{H} \rightarrow \gamma \gamma$ decay mode. For electrons (with energies from 20 to $250 \mathrm{GeV}$ ), incident within a $4 \times 4$ $\mathrm{mm}^{2}$ area about the point of maximum response, the mean values of the three contributions to the resolution, $\sigma(E) / E$, were $2.8 \% / \sqrt{E}$ (stochastic), $0.3 \%$ (inter-calibration) and $127 \mathrm{MeV} / E$ equivalent noise summed in a $3 \times 3$ crystal array [11].

\section{Conclusion}

The large number of photodetectors plus the difficulty of replacement after construction has provided a significant challenge during both the development and construction phases. We developed two extensive programmes of $100 \%$ acceptance testing combined with additional batch testing of the key components used in the production of the photodetectors. This should ensure that the failure, or unacceptable degradation in performance, of APD or VPT devices during the operational lifetime of CMS has been rendered extremely unlikely.

\section{Acknowledgements}

This work is presented on behalf of the whole CMS ECAL Group and the author thanks the technical and administrative staff at CERN and other CMS Institutes and acknowledges financial support from:

CERN, Department of Atomic Energy and Department of Science and Technology of India, U.S. Department of Energy, U.S. National Science Foundation, Croatian Ministry of Science, Education and Sport (under grant No. 023-0982887-3064), French CNRS/Institut de Physique Nucleaire et de Physique des Particules, French Commissariat a l'Energie Atomique, Greek General Secretariat for Research and Technology, Italian Istituto Nazionale di Fisica Nucleare, Federal Agency for Science and Innovations of the Ministry for Education and Science of the Russian Federation, Federal Agency for Atomic Energy of the Russian Federation, Russian Academy of Sciences, Ministry of Science and Technological Development of Serbia, Swiss Funding Agencies, Science and Technology Facilities Council (UK).

\section{References}

[1] S Chatrchyan, et al JINST 3 (2008) S08004.

[2] P. R. Lecoq, IEEE Trans.Nucl.Sci. 55 (2008) 1299.

[3] A.A. Annenkov, M.V. Korzhik, P. Lecoq, Nucl. Instr. and Meth. A 490 (2002) 30.

[4] Z. Antunovic, et al., Nucl. Instr. and Meth. A 537 (2005) 379.

[5] K.W. Bell, et al., IEEE Trans. Nucl. Sci. 51 (2004) 2284.

[6] K. Dieters, et al., Nucl. Instr. and Meth. A 461 (2001) 574.

[7] D. Renker, Nucl. Instr. and Meth. A 486 (2002) 164.

[8] Z Antunovic, et al, Nucl. Instr. and Meth. A 545 (2005) 139.

[9] K. Deiters et al. Nucl. Instr. and Meth. A 543 (2005) 549.

[10] P.R. Hobson, et al., Proc. ICATPP8, World Scientific, Singapore, 2004, pp. 635-639

[11] P. Adzic, et al, JINST 2 (2007) P04004. 